\title{
Mouse and human Notch-1 regulate mucosal immune responses
}

\author{
DR Mathern ${ }^{1,6}$, LE Laitman ${ }^{1,6}$, Z Hovhannisyan $^{1}$, D Dunkin ${ }^{2}$, S Farsio ${ }^{1}$, TJ Malik ${ }^{1}$, G Roda $^{1}$, A Chitre $^{1,3}$, \\ AC Iuga ${ }^{1,4}, G$ Yeretssian ${ }^{1}$, MC Berin ${ }^{5}$ and S Dahan ${ }^{1}$
}

The Notch-1 signaling pathway is responsible for homeostatic tight junction expression in vitro, and promotes barrier function in vivo in the RAG1-adoptive transfer model of colitis. In this study, we sought to determine the role of colonic Notch-1 in the lymphoepithelial crosstalk in health and disease. We utilized in vivo and in vitro knockdown to target the expression of Notch-1. We identified that epithelial Notch-1 is required for appropriate activation of intestinal epithelial cells at steady state and upon inflammatory stimulus. Notch-1 expression modulates mucosal chemokine and cytokine secretion, and FoxP3 and effector T-cell responses. We showed that epithelial Notch-1 controls the immune function of the epithelium through crosstalk with the nuclear factor-кB (NF-кB)/mitogen-activated protein kinase (MAPK) pathways that, in turn, elicits T-cell responses. Overall, epithelial Notch-1 bridges innate and adaptive immunity in the gut. Our findings highlight an indispensable role for Notch-1-mediated signaling in the intricate epithelial-immune crosstalk, and validate that epithelial Notch-1 is necessary and sufficient to support protective epithelial proinflammatory responses.

\section{INTRODUCTION}

The intestinal tract is exposed to a variety of food and foreign antigens from the external environment, discriminating between harmless and pathogenic antigens. The epithelium maintains a physical and immunological barrier to prevent invasion of luminal bacteria. Intestinal epithelial cells (IECs) act as sentinels for the immune system and nonprofessional antigen-presenting cells. ${ }^{1}$ The potential of IECs to promote regulatory $\mathrm{T}$ cells suggests a key role of IECs and the lymphoepithelial crosstalk in the maintenance of intestinal immune homeostasis. ${ }^{2,3}$ This crosstalk is often disrupted, as seen in patients with food allergy, inflammatory bowel disease, celiac disease, and colonic cancer, ${ }^{4}$ where alterations in cytokine secretion and regulatory T-cell function promote, rather than inhibit, inflammation. Further understanding of the nature of IEC/T-cell interactions during homeostasis will help decipher the mechanisms underlying the immune defects observed in disease states.
Notch signaling plays an indispensable role in gut formation from the earliest stages of primitive gut tube development, to the differentiation and proliferation of both epithelial stem cells and mature cells. ${ }^{5-8}$ Notch upregulates the expression of the transcriptional repressor Hairy and enhancer of split-1 (Hes-1) that inhibits the expression of Atonal homolog-1 (Atoh-1). Atoh-1expressing cells differentiate into secretory cells, whereas absorptive cells arise from Atoh-1-independent cells that express Hes-1 and ultimately Caudal-related homeoprotein-2 (CDX2). Beyond its critical roles in normal development and homeostasis of the gut, Notch signaling may be crucial to the maintenance of intestinal architecture and barrier function. Dysregulation of this pathway may alter the crosstalk between the intestinal epithelium and the underlying lymphocytes. We have shown that the absence of Notch-1 signaling is responsible for unbalanced tight junction (TJ) protein complexes and barrier dysfunction. Restitution of epithelial Notch-1 activation correlated with normal barrier function in the RAG1-adoptive transfer model of colitis. ${ }^{9,10}$

\footnotetext{
${ }^{1}$ Immunology Institute, Icahn School of Medicine at Mount Sinai, New York, New York, USA. ²Division of Pediatric Gastroenterology, Icahn School of Medicine at Mount Sinai, New York, New York, USA. ${ }^{3}$ Division of Experimental Medicine, San Francisco General Hospital, University of California, San Francisco, San Francisco, California, USA.

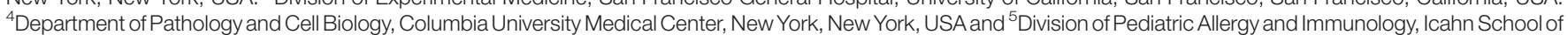
Medicine at Mount Sinai, New York, New York, USA. Correspondence: S Dahan (stephanie.dahan@mssm.edu)

${ }^{6}$ The first two authors contributed equally to this work.
} 
Notch signaling plays a critical role in the development of the immune system, both in thymic development and peripheral T-cell differentiation, ${ }^{11}$ but its role in peripheral T-cell activation is controversial and poorly defined. Notch signaling may activate T cells, and function as "signal 3" coactivation by induction of cytokine expression. ${ }^{12}$ Bacterial products can induce Notch ligand expression on dendritic cells and macrophages via Toll-like receptor stimulation, allowing $\mathrm{T}$-cell activation and polarization. ${ }^{13-15}$ Despite increasing reports on the role of Notch signaling in the development of the immune system and intestinal tract, there is no evidence of the role of Notch-1 in the intestinal epithelial immune response.

The purpose of this study was to determine the role of epithelial Notch-1 in the crosstalk between IECs and the underlying immune system. We show that epithelial Notch-1 is required for appropriate activation of IECs at steady state and upon inflammatory stimulus. Notch-1 expression modulates mucosal chemokine/cytokine secretion, and FoxP3 and effector $\mathrm{T}$-cell responses. Overall, our findings reveal a central and unique role for epithelial Notch-1 in bridging innate and adaptive immunity in the gut.

\section{RESULTS}

\section{Colonic Notch-1 controls TJ protein expression and barrier} integrity

RAG1-deficient mice exhibit impaired distal colonic IEC differentiation, defects in intestinal permeability, and absence of Notch-1 activation. ${ }^{9,16}$ Because Notch-1 controls epithelial differentiation, we postulated that Notch-1 might control the expression of TJ proteins, thereby regulating intestinal barrier function. Alterations in the expression and cellular localization of TJ proteins and decreased transepithelial resistance were observed in Notch-1 KD intestinal epithelial Caco-2 cells, supporting our hypothesis in vitro.

To further test this hypothesis in vivo, we knocked down Notch-1 in the distal colon of wild-type (WT) mice by intrarectal injection of liposome-delivered small interfering RNA (siRNA). To ensure that the siRNA effectively penetrated the distal colonic tissue, we used a DY547-tagged scrambled siRNA and tracked the fluorescence associated with the siRNA by confocal microscopy. As previously reported, intrarectal siRNA administration exclusively targeted the epithelial layer and did not penetrate the lamina propria (Supplementary Figure S1A online). ${ }^{17}$ Therefore, we used a Notch-1-targeted siRNA to deplete Notch-1 expression. Notch-1 or scrambled siRNA were administered on days 0 and 4 and mice were killed at days 2 and 5 . Notch-1 and downstream target gene mRNA expression was quantified by quantitative PCR (qPCR) in the distal colon. Notch-1 and CDX2 mRNA expression was significantly decreased in the Notch-1 siRNA-treated mice $(P=0.001$ and 0.007$)$, but Hes- 1 and Atoh-1 expressions were unchanged (Supplementary Figure S1B). Epithelial proliferation, apoptosis, and goblet cell loss did not show any differences between groups (data not shown). We investigated the expression of TJ genes by qPCR. Consistent with previous in vitro data, we observed a significant decrease in claudin- 5
$(P=0.008)$, but not claudin-8 or occludin mRNA expression in Notch-1 siRNA-treated mice (Figure 1a). Claudin-5 protein expression was confirmed by immunofluorescence (Figure 1b). In Notch-1 siRNA-treated mice, claudin-5 staining was significantly decreased in the intestinal crypts $(P=0.0003$; Figure 1c). The mRNA expression of these important genes was also evaluated in the transverse and proximal colon by qPCR and no differences were observed between groups (Supplementary Figure S1C). We performed Ussing chamber studies to assess the distal colonic permeability of siRNAtreated mice. fluorescein isothiocyanate-dextran $(3 \mathrm{kDa})$ was added to the luminal side, and its appearance in the serosal compartment was tracked every $30 \mathrm{~min}$ for $2 \mathrm{~h}$. Flux across the distal colon was increased fourfold in the Notch-1 siRNAtreated mice ( $P=0.03$; Figure 1d). Thus, Notch-1 regulates the expression of TJ proteins and colonic barrier function.

\section{Absence of colonic Notch-1 increases disease severity in experimental colitis}

Given that Notch-1 plays a role in the homeostatic regulation of epithelial gene expression and barrier function, we investigated the role of Notch-1 in inflammation. We addressed the implication of the Notch-1-inducing impaired barrier function using the chemically induced acute dextran sulfate sodium (DSS)-colitis, an epithelium-driven model of intestinal inflammation. Intrarectal scrambled (Sc-DSS) and Notch-1 (N-DSS) siRNA was administered on days $0,2,4$, and 6 , the $3 \%$ DSS regimen was started at day 4 and maintained until day 11 when all mice were killed. To ensure that the siRNA effectively penetrated the distal colonic tissue during inflammation, we tracked the fluorescence associated with the scrambled-DY547 siRNA by confocal microscopy. Intrarectal siRNA administration targeted the epithelial layer (Supplementary Figure S2A), but the fluorescence associated with the scrambledDY547 siRNA during DSS treatment seemed less intense than the scrambled-DY547 siRNA without DSS treatment (Supplementary Figure S1A). Notch-1 knockdown (KD) efficiency was confirmed as previously described. Notch-1 and Hes-1, but not Atoh-1, mRNA expression was decreased in N-DSS mice (Supplementary Figure S2A). Although the two groups exhibited similar weight loss at the end of the DSS treatment, N-DSS mice consistently exhibited greater weight loss $(P=0.02)$ at day 8 of the treatment (Figure 2a). In order to assess the molecular changes leading to this difference, we killed mice at day 8. N-DSS mice presented higher histological disease score $(P=0.01)$ in their distal colon (Figure $2 \mathbf{b})$. Histological analysis of the transverse and proximal colon showed no significant differences in colitis disease score (Supplementary Figure S2B). The siRNA treatment alone did not increase inflammation, suggesting that induction of inflammation is not caused by inherent properties of Notch-1 KD or siRNA administration (data not shown).

As the acute DSS model of colitis revealed a role for Notch-1 in inflammation, we investigated the implication of Notch-1 in the recovery DSS model of colitis. The siRNAs were administered on days $0,2,4,6,8$, and 10 and the $3 \%$ DSS regimen was 

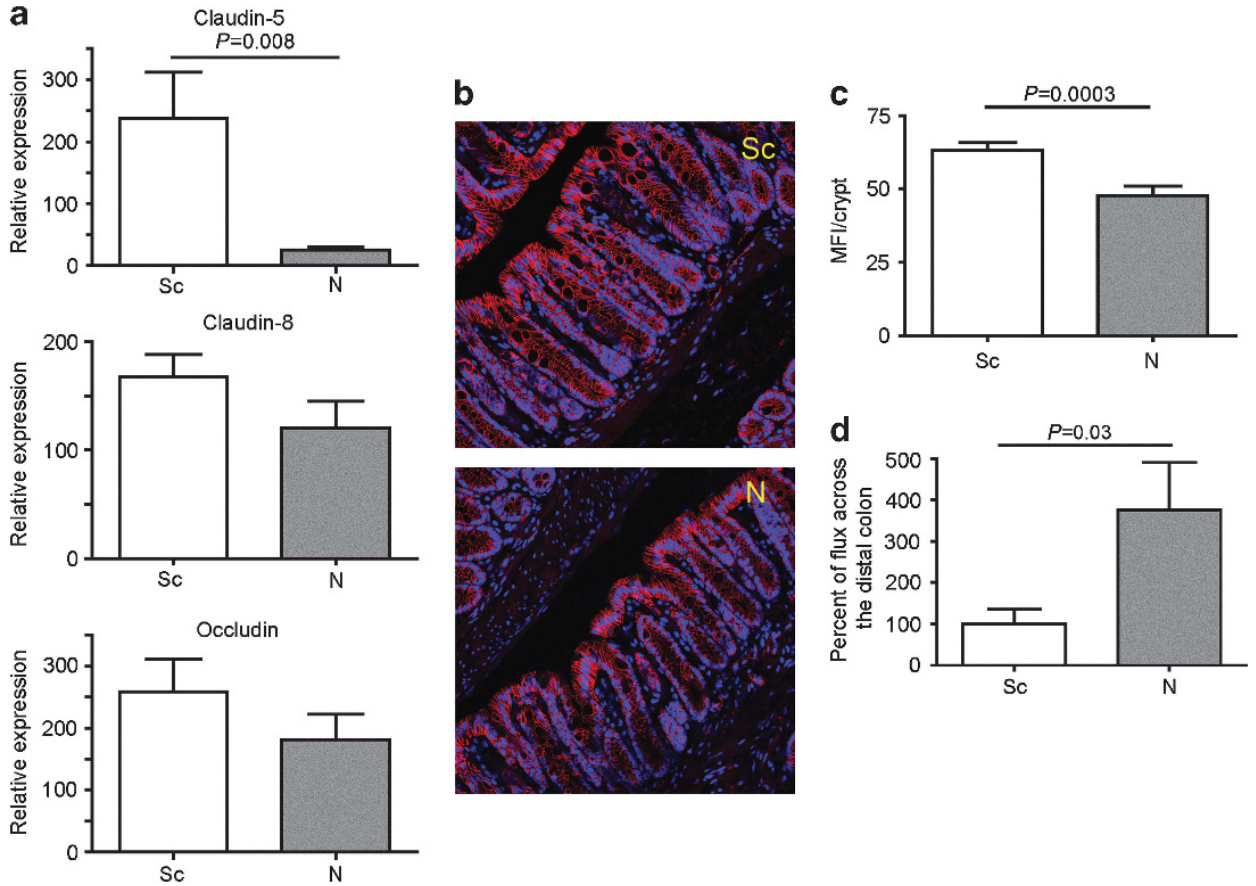

Figure 1 Effect of Notch-1 knockdown (KD) on intestinal barrier function in vivo. (a) Claudin-5, -8, and occludin mRNA expression assessed in the distal colon of scrambled (Sc) or Notch-1 (N) small interfering RNA (siRNA)-treated wild-type (WT) mice by quantitative PCR (qPCR). (b) Claudin-5 immunofluorescence staining (red) in the distal colon of Sc or N siRNA-treated WT mice. The nuclei were stained with 4',6-diamidino-2-phenylindole (DAPI; blue). Samples were analyzed with a Leica SP5-DM Confocal microscope. These data are representative of three experiments. (c) Quantification of mean fluorescence intensity (MFI) per crypt associated with claudin-5 staining using the Adobe Photoshop software. (d) Assessment of changes in paracellular permeability for small molecules. The distal colon was harvested and the paracellular permeability was measured (assessed by flux measurements of dextran-fluorescein isothiocyanate (FITC) from the mucosal to serosal compartment, measured by spectrofluorometry). Data represent the mean \pm s.e.m. of at least 9 mice/group. $P<0.05$ was considered statistically significant.

a

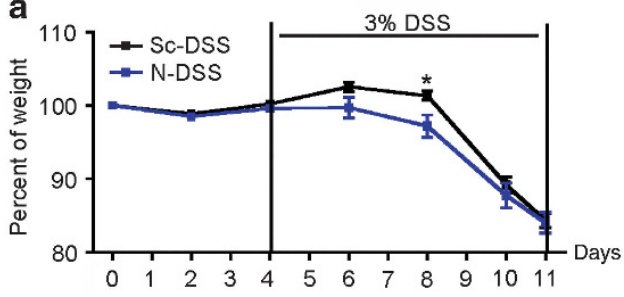

c

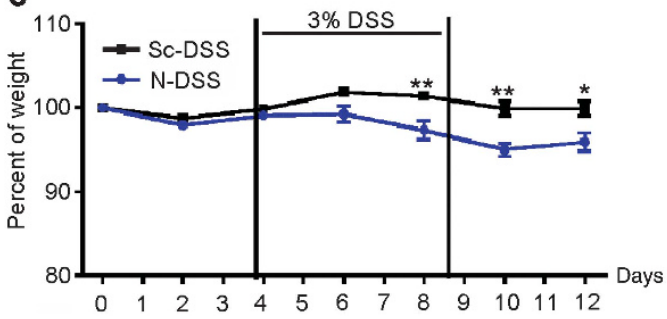

b

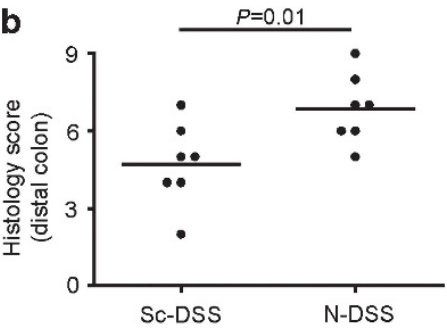

d

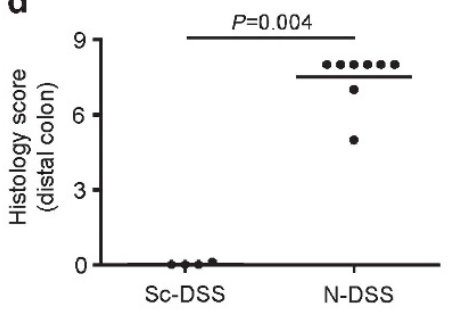

Figure 2 Effect of Notch-1 knockdown (KD) on dextran sulfate sodium (DSS)-induced colitis. (a) Percent body weight of mice treated with scrambled (Sc-DSS) or Notch-1 (N-DSS) small interfering RNA (siRNA) in conjunction with DSS treatment (acute model of DSS). (b) Histology score of the distal colon of Sc-DSS or N-DSS mice at day 8 of acute DSS treatment. (c) Percent body weight of Sc-DSS or N-DSS mice (recovery model of DSS). (d) Histology score of the distal colon of Sc-DSS or N-DSS mice at day 12 of recovery model of DSS. Data represent the mean \pm s.e.m. of 4-8 mice/group. $P<0.05$ was considered statistically significant.

maintained from days 4 to 9 . At day 9 , regular water replaced DSS until day 12 when mice were killed. N-DSS mice exhibited greater weight loss $(P=0.003)$ at day 8 (as observed above in
Figure 2a,c). This difference in weight between the two groups was sustained throughout the experiment $(P=0.003$ and 0.03 at day 10 and 12, respectively; Figure 2c), speaking for a role for 
Notch-1 in mucosal homeostasis. N-DSS mice presented higher histological disease score in the distal colon $(P=0.004$; Figure 2d). Thus, in vivo colonic Notch-1 deficiency leads to increased susceptibility to DSS-induced colitis, suggesting a paramount role for Notch-1 in intestinal inflammation and repair.

\section{Notch-1 controls the expression of colonic chemokines and immune cell responses}

The increased susceptibility of mice to DSS-induced colitis following treatment with Notch-1 siRNA led us to explore the expression of colonic chemokines by qPCR at day 8 (Figure 3 ). We observed significant decreases in CCL2 (MCP1) $(P=0.02)$, CCL20 $(P=0.01)$, and CXCL10 (IP-10) $(P=0.04)$ mRNA expression, and decreasing trends in CCL11, CXCL1 (KC), and CXLC2 (MIP2 $\alpha$ ) mRNA expression in N-DSS mice compared with Sc-DSS mice. Thus, a defect in Notch-1 expression dampens the colonic chemokine response to injury.

Impaired chemokine expression in the gut could lead to defects in the signaling cascades and compromise mucosal immune cell activation/migration and mesenteric lymph node (MLN) T-cell responses typically seen during inflammation.
We therefore evaluated the colonic and MLN expression of the chemokine receptors corresponding to the chemokines described above. We did not observe any difference in mRNA expression of these receptors (data not shown), speaking for a defect in mucosal immune cell activation rather than recruitment. Moreover, we did not observe differences in cellularity in the MLNs between the two groups (data not shown). We then investigated lymphocyte responses in the draining MLNs. MLN cell suspensions from N-DSS and ScDSS mice were stimulated with anti-CD3/CD28 antibodies for 3 days and inflammatory cytokines were quantified by Cytometric Bead Array (CBA) (Figure 4). Interleukin (IL)2 secretion was similar between both groups, suggesting that T-cell activation was not compromised (data not shown). However, we detected a significant decrease in IL-10 $(P=0.03)$, interferon- $\gamma($ IFN $\gamma ; P=0.05)$, and tumor necrosis factor- $\alpha$ (TNF $\alpha ; P=0.04)$ secretion, and decreasing trends in IL-6 and IL-17A production from MLN cells derived from N-DSS mice. Thus, a defect in colonic Notch-1 expression affects the expression of T-cell cytokines in the MLNs in response to gut mucosal injury.
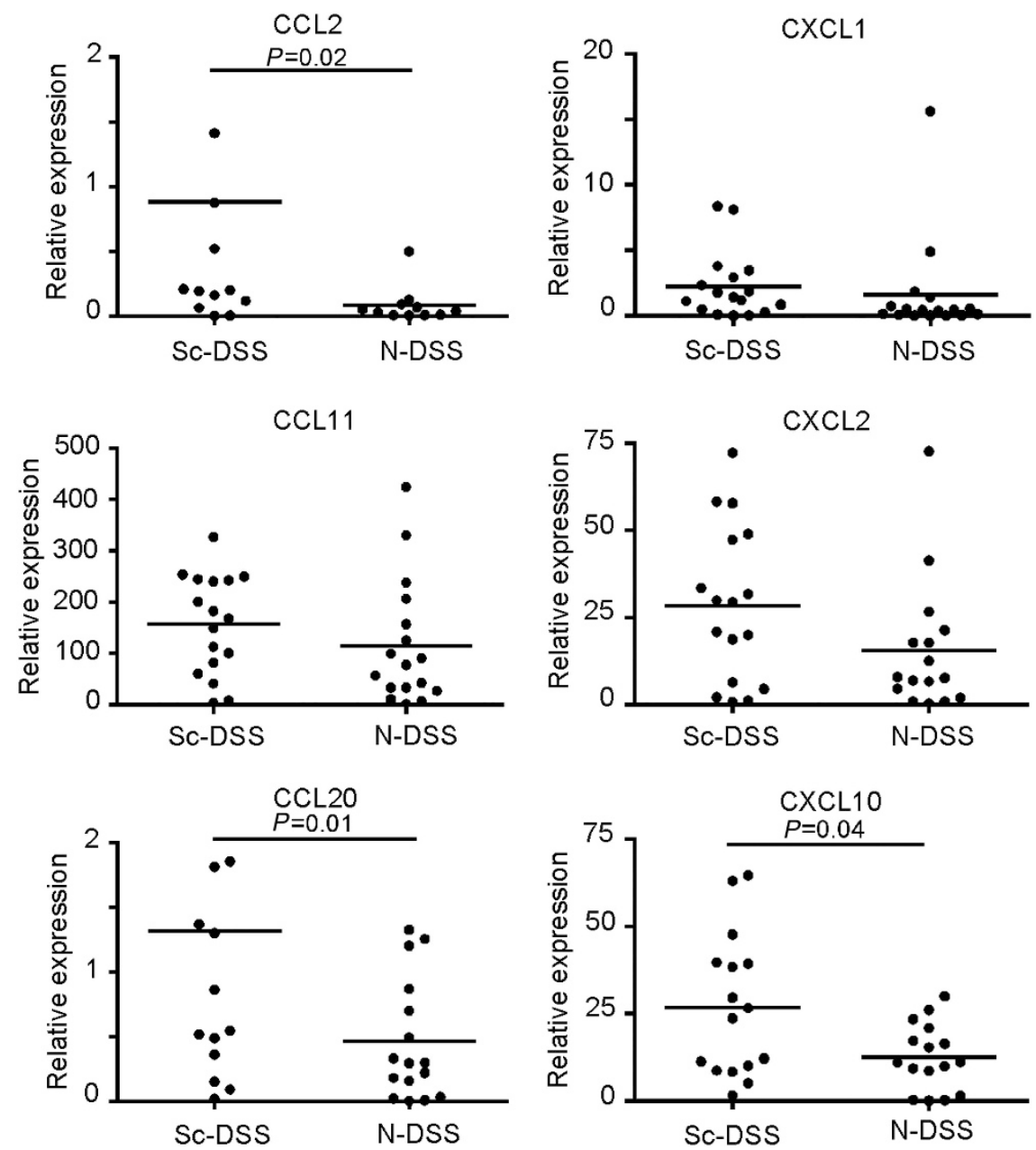

Figure 3 Effect of Notch-1 knockdown (KD) on colonic chemokine and cytokine expression during colonic injury. CCL2, 11, 20, and CXCL1, 2, and 10 mRNA expression was assessed in the distal colon of scrambled dextran sulfate sodium (Sc-DSS) or Notch-1 DSS (N-DSS) mice by quantitative PCR (qPCR). Data represent individual points and average mean of each group; $n=11-17$ mice/group. $P<0.05$ was considered statistically significant. 

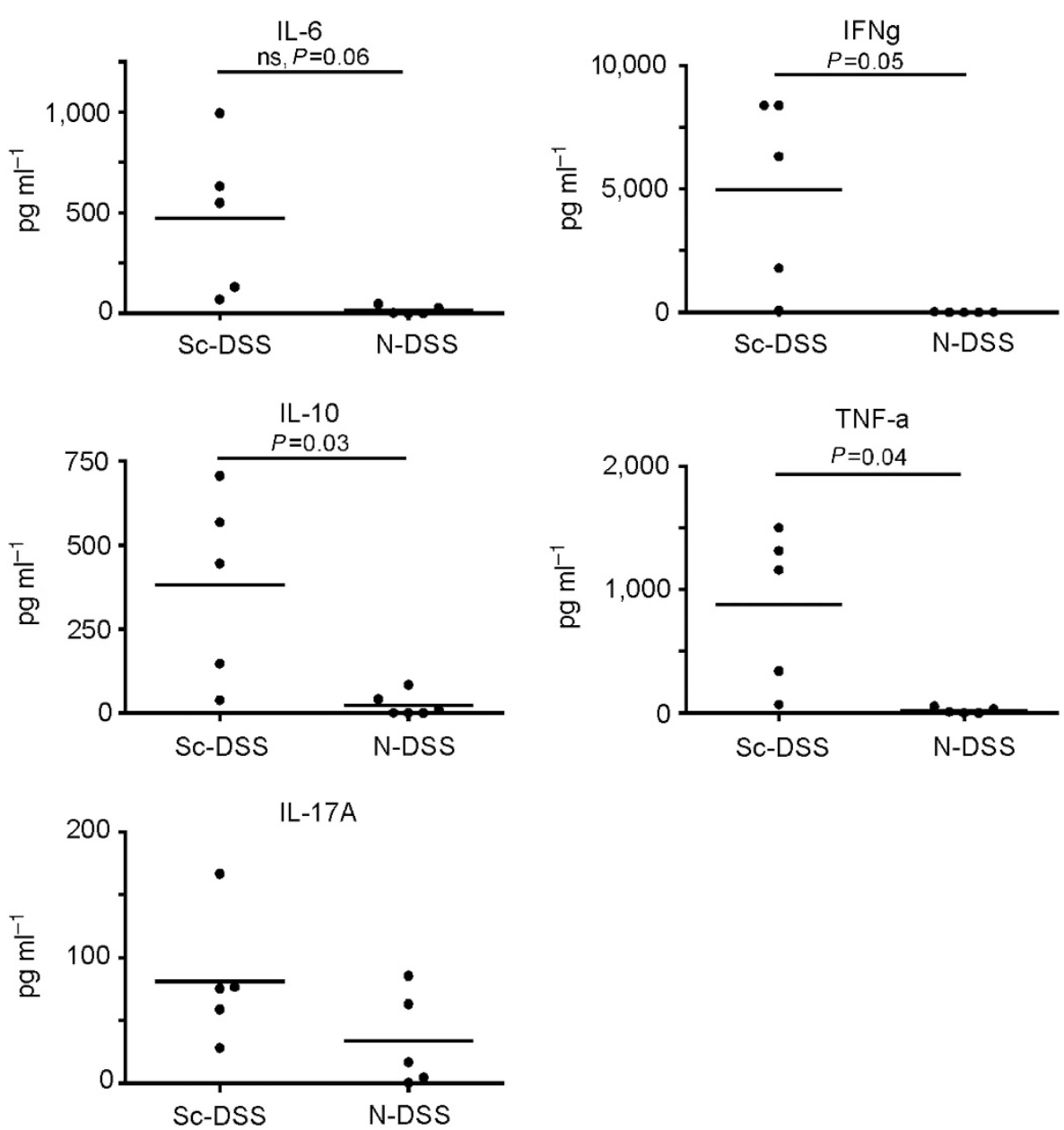

Figure 4 Effect of Notch-1 knockdown (KD) on effector T-cell cytokine secretion. Interleukin (IL)-6, -10, -17A, interferon- $\gamma$ (IFN $\gamma$ ), and tumor necrosis factor- $\alpha$ (TNF $\alpha$ ) cytokine secretion was assessed in the culture supernatant of anti-CD3/CD28-stimulated mesenteric lymph node (MLN) cells of scrambled dextran sulfate sodium (Sc-DSS) or Notch-1 DSS (N-DSS) mice by Cytometric Bead Array (CBA). Each data point represents a pool of three mice and the average mean of each group. $P<0.05$ was considered statistically significant.

IL-10 generally characterizes the regulatory T-cell signature in the gut. ${ }^{18}$ The decreased IL-10 secretion prompted us to explore the expression of FoxP3 + cells in both the distal colon and the MLNs. FoxP3 protein expression was assessed by immunofluorescence (Figure 5a). In N-DSS mice, FoxP3 staining was decreased in the lamina propria. The frequency of FoxP $3+\mathrm{CD} 4+\mathrm{T}$ cells was also determined in the MLNs by flow cytometry. Although the cellularity and the viability were identical between the two groups (data not shown), we observed a significant decrease in the frequency of FoxP3 + CD4 + $\mathrm{T}$ cells $(P=0.004)$ in N-DSS vs. Sc-DSS mice (Figure $\mathbf{5 b}$ ).

Based on the impaired effector T-cell cytokine profile and in particular IFN $\gamma$ secretion, we determined the frequency of $\mathrm{T}$ helper type 1 (Th1) cells in the MLNs by flow cytometry. We observed a decrease in the frequency of IFN $\gamma+\mathrm{CD} 4+$ T cells $(P=0.03)$ in $\mathrm{N}$-DSS mice compared with Sc-DSS mice (Figure 5c).

Altogether, these data support a critical role for colonic Notch-1 in both innate and adaptive immune responses to DSS-induced injury.

\section{Notch-1 controls the expression of proinflammatory cytokines in Caco-2 cells}

Thus far, the epithelial Notch-1 signaling pathway has only been explored in the context of epithelial proliferation and differentiation. There is growing evidence supporting a role for Notch within the immune compartment. ${ }^{11-15}$ However, the mechanism by which epithelial Notch-1 enables IECs to function as immune cells remains unknown. To investigate this, we used Caco-2 cell lines stably transfected with control and Notch-1 short hairpin RNA as previously developed in our lab ${ }^{9}$ and analyzed chemokine/cytokine gene expression by qPCR (Figure 6a and Supplementary Figure S3). Concomitantly, Caco-2 supernatants were harvested for quantification of cytokine levels by CBA (Figure $6 \mathbf{b}$ ). We observed reduced basal mRNA expression of IL-1 $\beta(P=0.01)$, IL-6 $(P=0.02)$, IL-8 $(P=0.01)$, and TNF $\alpha(P=0.01)$, and decreased IL- 8 secretion in Notch-1 KD Caco-2 cells $(P=0.05)$.

Caco- 2 cells were then stimulated with TNF $\alpha$ to create an inflammatory environment in vitro. Consistent with our in vivo model, TNF $\alpha$-stimulated Notch-1 KD Caco-2 cells exhibited 

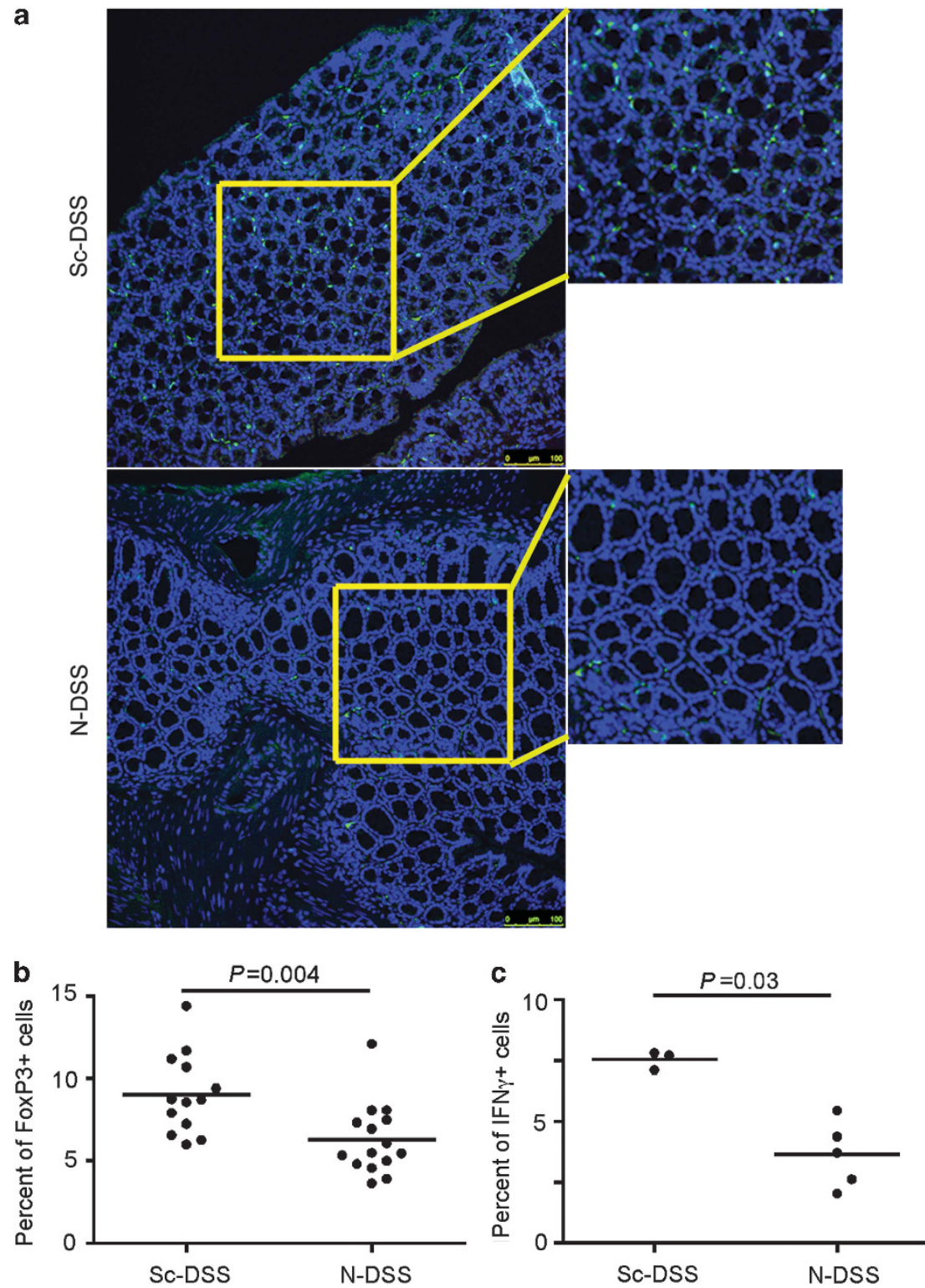

Figure 5 Effect of Notch-1 knockdown (KD) on regulatory and effector T cells during dextran sulfate sodium (DSS)-induced colitis. (a) FoxP3 immunofluorescence staining (green) of the distal colon of scrambled DSS (Sc-DSS) or Notch-1 DSS (N-DSS) mice. The nuclei were stained with 4',6-diamidino-2-phenylindole (DAPI; blue). An enlarged representation of the yellow rectangle is shown. Samples were analyzed with a Leica SP5-DM Confocal microscope. These data are representative of three experiments. (b) Flow cytometry analysis of FoxP3 + CD4 + T mesenteric lymph node (MLN) cells of Sc-DSS or N-DSS mice. (c) Flow cytometry analysis of interferon- $\gamma$ (IFN $\gamma$ ) + CD4 + T MLN cells of Sc-DSS or N-DSS mice. Each data point represents a pool of three mice and the average mean of each group. $P<0.05$ was considered statistically significant.

significantly decreased IL-1 $\beta(P=0.009)$, IL-6 $(P=0.04)$, IL-8 $(P=0.006)$, CCL20 $(P=0.03)$, and CXCL10 $(P=0.05)$ (Figure 6a and Supplementary Figure S3), but not TNF $\alpha$ and CCL2 mRNA expression, and decreased IL-8 secretion $(P=0.001 ;$ Figure $6 \mathbf{b})$. This finding supports the critical role of Notch-1 in IEC immune cell function and its implication in regulating a proper epithelial proinflammatory response.

The gene expression of IL-8 as well as other cytokines is under the transcriptional regulation of the activator protein $1 /$ nuclear factor- $\kappa \mathrm{B}(\mathrm{NF}-\kappa \mathrm{B})$ axis. ${ }^{19}$ Thus, we investigated the potential crosstalk between Notch-1 and mitogen-activated protein kinase (MAPK)/NF- $\kappa \mathrm{B}$ signaling pathways by western blot analyses with antibodies against phospho-ERK1/2, phospho-p38, phospho-JNK, and IкB- $\alpha$. We observed decreased p38 activation upon TNF $\alpha$ stimulation of Notch-1 KD Caco-2 cells (Figure 6c), but we did not detect differences for extracellular signal-regulated kinases 1 and 2 (ERK1/2) and c-Jun N-terminal kinase (JNK; data not shown). However, we noticed an increase in basal IкB- $\alpha$ expression in the Notch-1 KD Caco-2 cells without further differences in I $\mathrm{K} B-\alpha$ degradation upon TNF $\alpha$ stimulation. These results speak for a crosstalk between Notch-1 and MAPK/NF- $\kappa B$ signaling 

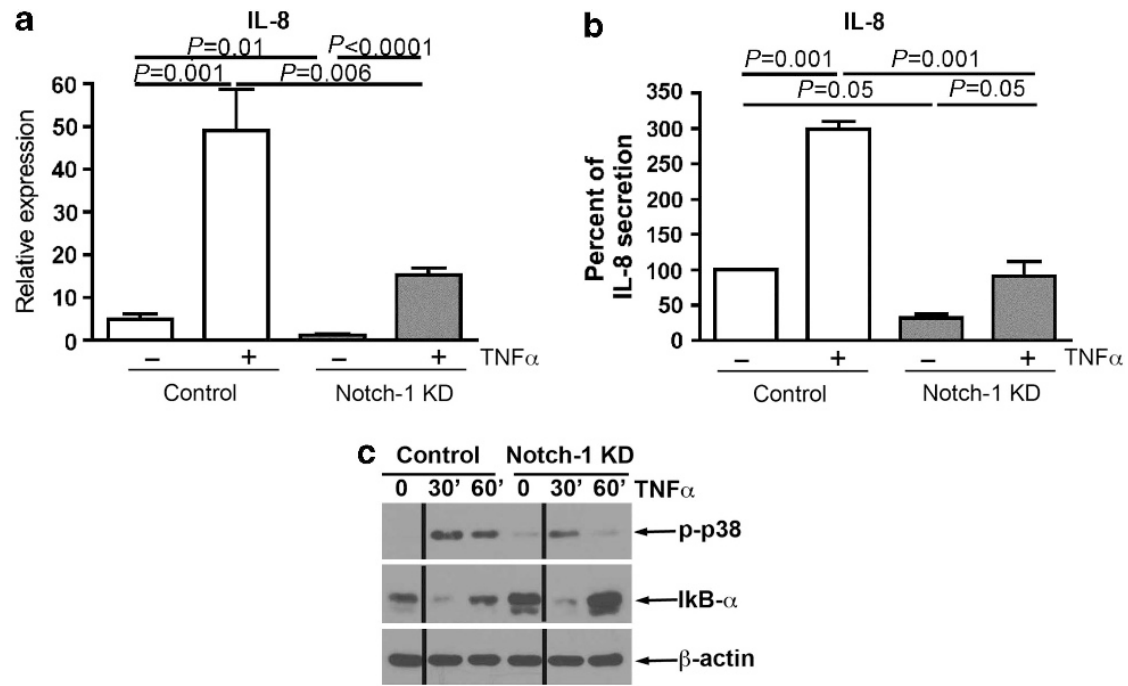

Figure 6 Effect of Notch-1 knockdown (KD) on epithelial proinflammatory responses. (a) Interleukin-8 (IL-8) mRNA expression was assessed in control and Notch-1 short hairpin (shRNA)-treated Caco-2 cells. Cells were incubated with or without tumor necrosis factor- $\alpha$ (TNF $\alpha$ ) for $4 \mathrm{~h}$. (b) IL-8 secretion of cell supernatants from control or Notch-1 shRNA-treated Caco-2 cells was quantified after a 2-h TNF $\alpha$ pulse followed by a 2-h rest by Cytometric Bead Array (CBA). Data represent the mean \pm s.e.m. of at least four independent experiments. $P<0.05$ was considered statistically significant.

(c) Immunoblotting for $\mathrm{p}-\mathrm{p} 38, \mathrm{I} \mathrm{K} \mathrm{B}-\alpha$, and $\beta$-actin in lysates obtained from TNF- $\alpha$-treated control or Notch-1 shRNA Caco-2 cells (representative of three experiments).

pathways. Moreover, these data confirm the in vivo observations (Figure 2) and underscore the importance of Notch-1 in $\mathrm{TNF} \alpha$-mediated epithelial inflammatory responses.

\section{Epithelial Notch-1 controls T-cell responses}

A defect in Notch-1 leads to inappropriate IEC activation in vitro, and decreased effector T-cell cytokines and regulatory $\mathrm{T}$ cells in vivo. However, a role for epithelial Notch-1 in enabling T-cell function has not been defined. Peripheral blood mononuclear cells (PBMCs) from healthy individuals were stimulated with anti-CD3/CD28 Dynabeads in the presence of TNF $\alpha$-pulsed control or Notch-1 KD Caco- 2 cell supernatants. The percentage of FoxP $3+$ and IFN $\gamma+$ T cells was analyzed by flow cytometry (Figure 7a). PBMC supernatants were collected to quantify effector T-cell cytokine secretion by CBA (Figure $7 \mathbf{b}$ ). Although the cellularity and the viability were identical between the two groups (data not shown), supernatants derived from Notch-1 KD Caco-2 cells demonstrated a reduced ability to promote FoxP3 $+(P=0.003)$ and IFN $\gamma+$ $(P=0.01)$ CD 4 T cells compared with control Caco- 2 cells. We then analyzed effector cytokine production. Consistent with our in vivo studies, supernatants from Notch-1 KD Caco-2 cells induced significantly lower IL-6 $(P=0.02)$ and IFN $\gamma(P=0.04)$ secretion from PBMCs, and decreased IL-10 production $(P=0.058)$. IL-2 production remained unchanged (data not shown). These results suggest that the decrease observed in effector and regulatory cell frequencies as well as their corresponding cytokine levels is a direct effect of epithelial Notch-1 expression and not a consequence of cell death or hyporesponsiveness. Taken together, these data emphasize the critical role of epithelial Notch-1 in controlling the intricate lymphoepithelial crosstalk.

\section{DISCUSSION}

There is growing evidence for the important role of the Notch pathway within the immune compartment. ${ }^{11-15}$ The intestinal epithelium is a critical component of the mucosal immune response. The interplay between the epithelium and the underlying immune system requires bidirectional communication, eliciting T-cell responses in homeostasis and in disease, as well as driving IEC differentiation and function. In this study, we investigated the role of epithelial Notch-1 in controlling the immune function of the epithelium.

The crosstalk between the Notch- 1 and NF- $\kappa B$ pathways has been documented in macrophages and dendritic cells, ${ }^{14,20,21}$ but unexplored in the intestinal epithelium, a cellular compartment known to fully rely on Notch-1 for its embryonic development. ${ }^{7}$ Most studies have used either pharmacological $\gamma$-secretase inhibitors or genetically modulated RBP-J (Recombination signal Binding Protein for Ig $\kappa \mathrm{J}$ region) expression, a downstream regulator of Notch-1 activation. The use of $\gamma$-secretase inhibitors favors Atoh-1 expression (because of derepression of Atoh-1 from loss of its negative regulator, Hes-1) and induces goblet cell metaplasia. ${ }^{22}$ Furthermore, knocking out RBP-J in the intestinal epithelium in vivo leads to goblet cell hyperplasia, increased permeability, and chronic colitis characterized by Th17 cells. ${ }^{23}$ Thus, $\gamma$-secretase inhibitors and the epithelial RBP-J knockout mouse model have the same end point as both exhibit goblet cell abnormalities. To avoid tipping epithelial cell differentiation towards the goblet cell phenotype, we aimed at globally downregulating Notch-1 expression.

In this study, we focused our experiments on the distal colon because our previous work showed striking differences in the distal colon of RAG1-deficient mice compared with WT mice. 

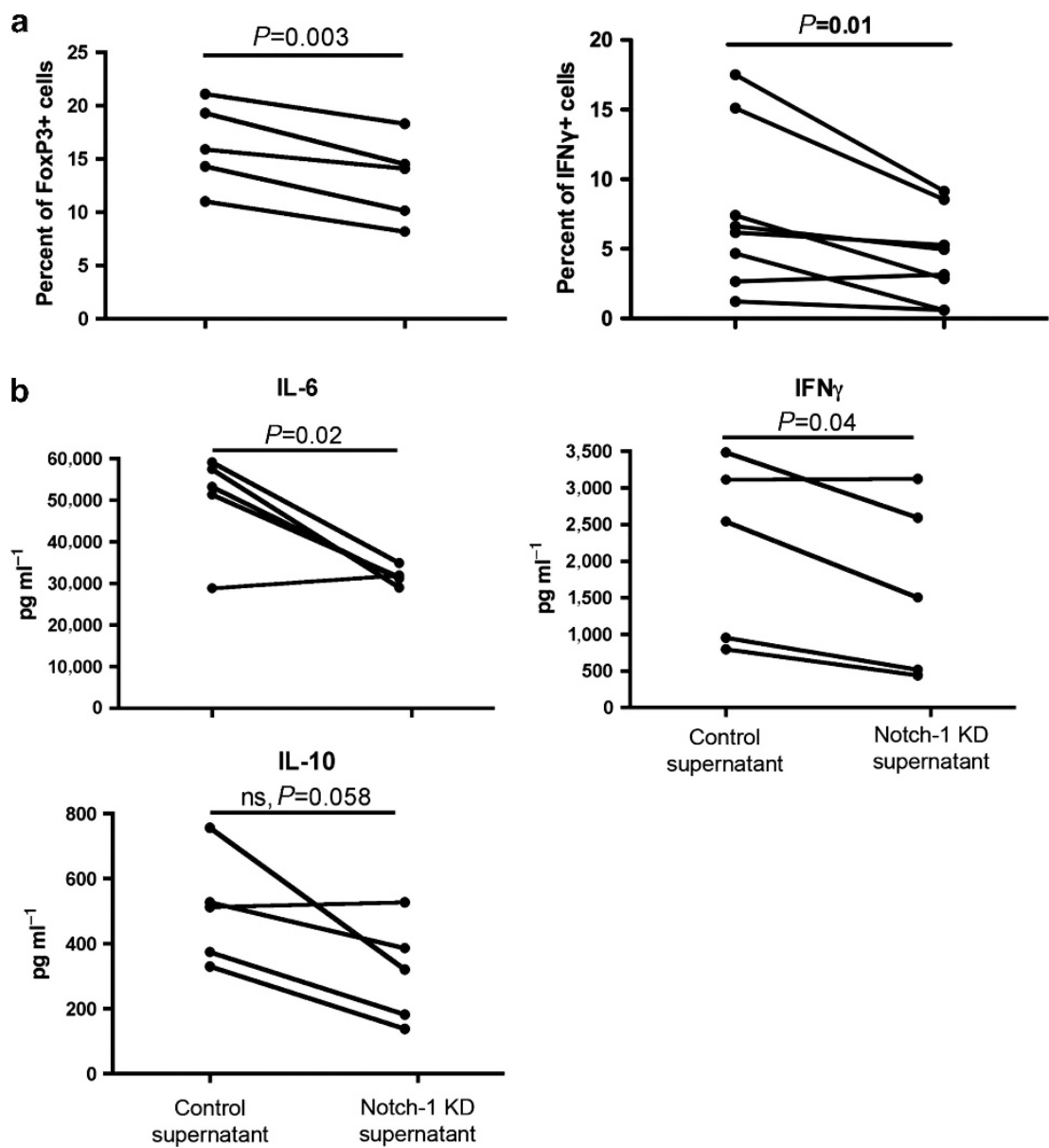

Figure 7 Effect of tumor necrosis factor- $\alpha(T N F)$ )-stimulated Notch-1 knockdown (KD) Caco-2 cell on peripheral blood T-cell subsets. (a) Flow cytometry analysis of FoxP3 + CD4 T cells and interferon- $\gamma$ (IFN $\gamma$ ) + CD4 T cells from anti-CD3/CD28-stimulated peripheral blood mononuclear cells (PBMCs) from healthy individuals incubated with TNF $\alpha$-stimulated control short hairpin (shRNA) or Notch-1 KD Caco-2 cell supernatants. (b) Interleukin (IL)-6, -10, and IFN $\gamma$ cytokine secretion assessed by Cytometric Bead Array (CBA) from culture supernatants of anti-CD3/CD28-stimulated PBMCs from healthy individuals incubated with TNF $\alpha$-stimulated control shRNA and Notch-1 KD Caco-2 cell supernatants; $n=5-8$ individuals. $P<0.05$ was considered statistically significant.

Activation of the Notch-1 signaling pathway and epithelial permeability of the distal colon were dysregulated in RAG1deficient mice compared with WT mice. ${ }^{9}$ These differences were not as dramatic in other parts of the colon. The inflammatory environment of the distal colon is most likely very different from other sites of the intestinal tract, which could explain the correlation between the role of Notch-1 not only in IEC differentiation, but also in IEC function. We speculated that a complex site-specific interplay exists between the flora, the epithelium, and the underlying immune system.

Using in vitro and in vivo silencing approaches, we have elucidated a critical role for Notch-1 in triggering appropriate IEC activation and subsequent innate and adaptive immune responses. As previously described in vitro, ${ }^{9}$ we have shown that Notch-1 controls colonic barrier function in vivo. Although other cell types within the colonic epithelium express Notch-1, ${ }^{24}$ we have demonstrated in this study that the lack of epithelial Notch-1 expression affects basal levels of epithelial cytokine expression, especially IL-8, and prevents their increased expression upon TNF- $\alpha$ stimulation. The gene expression of IL- 8 is under the transcriptional regulation of the activator protein $1 / \mathrm{NF}-\kappa \mathrm{B}$ axis. ${ }^{19}$ Here, we showed a crosstalk between Notch-1 and MAPK/NF- $\kappa B$ pathways. It has been previously described that interactions between these signaling cascades occur in several cancer processes, including leukemia, colon, and breast cancers. ${ }^{25-27}$ The MAPK and NF$\kappa \mathrm{B}$ pathways share some common upstream molecules; the best described is the adaptor molecule MyD88. ${ }^{28}$ MyD88- / mice are more susceptible to DSS-induced colitis because of defects in immune cell recruitment and activation, leading to breached barrier and subsequent increased bacterial translocation. ${ }^{29,30}$ A defect in CXCL2 (MIP2 $\alpha$ ) secretion has been associated with decreased neutrophil recruitment and increased disease susceptibility. Moreover, knocking out MyD88 specifically in the intestinal epithelium increased the susceptibility to DSS-induced colitis, by causing defects in epithelial immune gene expression, resulting in a compromised host response to epithelial injury and increased colonic inflammation. ${ }^{31}$ 
In addition, IEC-intrinsic NF- $\kappa \mathrm{B}$-dependent gene expression was shown to be a critical regulator of antigen-presenting cellmediated immune responses in the gut and T-cell activation. ${ }^{32}$ Here we have shown a decrease in the expression of colonic chemokines/cytokines following Notch-1 silencing and a crosstalk between MAPK/NF- $\mathrm{KB}$ and Notch-1 signaling pathways. Overall, our in vivo model and ex vivo cocultures emphasize the role of the crosstalk between the epithelium and innate/adaptive immune responses. This explains the increased susceptibility to colitis of the N-DSS mice. Our findings suggest that Notch-1 signaling in the intestinal epithelium is not only homeostatic, but also a protective component of the host response to inflammation by acting on the MAPK/NF- $\kappa B$ axis and TJs.

In this report, we have shown that barrier function could be altered by a molecular mechanism independent of inflammation. Barrier dysfunction is a key component of many diseases in the gut, including food allergy, inflammatory bowel disease, celiac disease, and colon cancers. ${ }^{4}$ In some cases, the development of these diseases has been associated with disruptions in intestinal barrier elements, leading to permeability defects and aberrant responses of the underlying immune system. ${ }^{33}$ In the colon, cancer is caused by genetic alterations inducing epithelial cell transformation. ${ }^{34}$ Soler et al. ${ }^{35}$ in 1999 showed increased intestinal permeability of human colon cancers. More importantly, increased TJ permeability was found in human colon polyps, especially in those that were associated with colon cancer. More recently, barrier deterioration has been shown to induce colon carcinogenesis via increased bacterial translocation. ${ }^{36}$ Although we cannot rule out a role for the microbiota and potential dysbiosis in our in vivo model, our in vitro experiments clearly demonstrated a lymphoepithelial crosstalk governed by epithelial Notch-1 signaling.

The crosstalk between the epithelium and the underlying intestinal immune system has been underestimated for many years, neglecting the epithelial component of the mucosal immune system. Increasing evidence points to the important role of the IECs in homeostasis and in disease. Diverse reports have emphasized the key role of the IECs in promoting CD8 + suppressor T cells. ${ }^{2,3,37,38}$ In this work, we have furthered these observations and demonstrated that IECs can elicit effector and regulatory T-cell responses. We have shown that loss of Notch-1 induces increased intestinal permeability, dampens proinflammatory signals, and leads to inappropriate T-cell function.

In conclusion, our findings highlight an indispensable role for Notch-1-mediated signaling in the intricate epithelialimmune crosstalk, and validate for the first time that epithelial Notch-1 is necessary and sufficient to support protective epithelial proinflammatory responses.

\section{METHODS}

Mice. C57BL/6 WT females (Jackson Laboratories, Bar Harbor, ME) were housed in the Icahn School of Medicine at Mount Sinai specific pathogen-free barrier facility. All procedures were approved by Institutional Animal Care and Use Committee.
In vivo Notch-1 knockdown and DSS-induced colitis. Notch-1 or control DY547-labeled scrambled siRNA (Dharmacon, Thermo Scientific, Waltham, MA) were prepared as described previously. ${ }^{17,39}$ The siRNAs were administered intrarectally to anesthetized mice on days 0 , $2,4,6,8$, and 10. DSS (MP Biomedicals, Solon, $\mathrm{OH}$ ) was dissolved into distilled water at $3 \% \mathrm{wt} / \mathrm{vol}$ concentration. DSS or distilled water was administered on days $4-8,4-9$, or 4-11 of siRNA treatment. Weights were recorded every 2 days. Colons were collected for RNA extraction, histology, and Ussing chamber experiments. Histology was scored for mucosal, submucosal, and muscularis damage by a pathologist blinded to the treatment group. ${ }^{40}$ MLNs were harvested.

Mouse MLN stimulation. MLN cells were cultured on a plate precoated with anti-CD3/CD28 antibodies (eBioscience, San Diego, CA) for 3 days. Supernatants were analyzed by CBA with the Mouse Th1/Th2/Th17 kit (BD Biosciences, San Jose, CA). All CBA experiments were run on a LSR Fortessa (BD Pharmingen, Mississauga, ON, Canada), and analyzed using FlowJo software (Treestar, Ashland, OR).

Caco-2 cell culture. Control short hairpin RNA and Notch-1 KD Caco-2 lines were generated and maintained as previously described. ${ }^{9}$ Cells were stimulated with $10 \mathrm{ng} \mathrm{ml}^{-1}$ TNF $\alpha$ (R\&D Systems, Minneapolis, MN) for $4 \mathrm{~h}$ and processed for mRNA extraction. Supernatants from cells pulsed with TNF $\alpha$ for $2 \mathrm{~h}$ and rested for additional $2 \mathrm{~h}$ were collected, analyzed using the Human IL-8 CBA Flex kit (BD Biosciences), and frozen at $-80^{\circ} \mathrm{C}$ for PBMC culture.

Western blot analysis. Western blots were performed as described using anti-IкB- $\alpha$, anti-phospho-p38, and anti- $\beta$-actin antibodies (Cell Signaling, Danvers, MA). ${ }^{16}$

Human PBMC culture. PBMCs from healthy donors $(n=5-8)$ were cultured with anti-CD3/CD28 human T-Activator Dynabeads (Life Technologies, Carlsbad, CA) in Caco- 2 cell supernatant for 4 days. Supernatants were analyzed with the CBA Human Th1/Th2/Th17 kit (BD Biosciences) on a LSR Fortessa. Cells were processed for flow cytometry analyses.

Quantitative PCR. RNA from homogenized colonic tissues or Caco-2 cells was extracted using Trizol (Life Technologies). RNA was reverse transcribed using the qScript cDNA system (Quanta Biosciences, Gaithersburg, MD). Mouse Notch-1, Hes-1, Atoh-1, claudin-5, claudin- 8 , occludin, and actin, and human IL-1 $\beta$, IL-6, IL-8, TNF $\alpha$, CCL2, CCL20, CXCL10, and actin mRNA expression was quantified using the Solaris qPCR Gene Expression Assay system accompanied by the Solaris probes (Thermo Scientific). Mouse CCL2, CCL11, CCL20, CXCL1, CXCL2, CXCL10, and L32 mRNA expression was quantified using the SYBR Advantage qPCR system (Clontech Laboratories, Mountain View, CA) (Supplementary Table S1 online for primer sequences). qPCR experiments were performed and analyzed using the Applied Biosystems Viia7 Real-Time PCR platform (Life Technologies). Transcripts were assayed in duplicate, and the median CT values were used to calculate the relative expression as ${ }_{2-}{ }^{\Delta \mathrm{CT}}$ where the $\Delta \mathrm{CT}$ corresponds to mean of $\left(\mathrm{CT}_{\mathrm{Target}}\right.$ gene $\left.-\mathrm{CT}_{\mathrm{Actin}}\right)$.

Fluorescence microscopy. Frozen sections of distal colon from mice treated with DY547-tagged scrambled siRNA were counterstained with 4',6-diamidino-2-phenylindole (Life Technologies). Immunofluorescence detection of paraffin-embedded colonic tissue sections was performed using anti-claudin-5 with anti-rabbit IgG-Alexa 594 (Life Technologies), anti-FoxP3 (eBioscience) with anti-rat IgG-Alexa488 (Life Technologies), and counterstained with 4,6-diamidino-2-phenylindole. ${ }^{9}$ Slides were examined using a Leica SP5-DM confocal microscope (Leica, Bannockburn, IL). Quantification of mean fluorescence intensity was assessed using the Adobe Photoshop software (San Jose, CA).

Ussing chamber. Permeability studies were performed using fluorescein isothiocyanate-dextran (Sigma-Aldrich, St Louis, MO) in Ussing chambers (Physiological Instruments, San Diego, CA). ${ }^{9}$ 
Flow cytometry. Cells were stimulated with $50 \mathrm{ng} \mathrm{ml}^{-1}$ phorbol-12myristate-13-acetate (Sigma-Aldrich), $1 \mu \mathrm{g} \mathrm{ml}^{-1}$ ionomycin (SigmaAldrich), and GolgiPlug (BD Biosciences) for $4 \mathrm{~h}$. Cells were stained with anti-CD3, anti-CD4, anti-CD8 (human PBMCs only), anti-CD45 (mouse MLN cells only), and anti-IFN $\gamma$ antibodies (eBioscience), with Aqua live/dead fixable stain (Life Technologies), and with an antiFoxP3 antibody using the FoxP3 staining buffer set (eBioscience). As phorbol-12-myristate-13-acetate/ionomycin treatment of human PBMC downregulates surface CD4 expression, we stained for surface CD8 when assessing IFN $\gamma$ intracellular expression. Cells were run on a LSR Fortessa.

Statistical analysis. Statistical significance was determined by oneway analysis of variance (when there were more than two groups), nonparametric $t$-test (two groups only), or paired $t$-test (paired human PBMC samples). A value of $P<0.05$ was considered significant. All analyses were performed and data were graphed with Prism software (GraphPad, La Jolla, CA).

SUPPLEMENTARY MATERIAL is linked to the online version of the paper at http://www.nature.com/mi

\section{ACKNOWLEDGMENTS}

We thank Lloyd Mayer for his constant support. Tissue sectioning and microscopy analyses were performed at the MSSM-Histology and MSSMMicroscopy Shared Resource Facilities, respectively. SD, DD, and ZH were supported by the Crohn's and Colitis Foundation of America, and GY by the Helmsley Charitable Trust Fund.

\section{DISCLOSURE}

The authors declared no conflict of interest.

c) 2014 Society for Mucosal Immunology

\section{REFERENCES}

1. Dahan, S., Roth-Walter, F., Arnaboldi, P., Agarwal, S. \& Mayer, L. Epithelia: lymphocyte interactions in the gut. Immunol. Rev. 215, 243-253 (2007).

2. Allez, M., Brimnes, J., Dotan, I. \& Mayer, L. Expansion of CD8 + Tcells with regulatory function after interaction with intestinal epithelial cells. Gastroenterology 123, 1516-1526 (2002).

3. Allez, M., Brimnes, J., Shao, L., Dotan, I., Nakazawa, A. \& Mayer, L. Activation of a unique population of CD8 $(+)$ T cells by intestinal epithelial cells. Ann. NY Acad. Sci. 1029, 22-35 (2004).

4. Shaykhiev, R. \& Bals, R. Interactions between epithelial cells and leukocytes in immunity and tissue homeostasis. J. Leukoc. Biol. 82 1-15 (2007).

5. Crosnier, C., Vargesson, N., Gschmeissner, S., Ariza-McNaughton, L., Morrison, A. \& Lewis, J. Delta-Notch signalling controls commitment to a secretory fate in the zebrafish intestine. Development 132, 1093-1104 (2005).

6. Kopan, R. \& llagan, M.X. The canonical Notch signaling pathway: unfolding the activation mechanism. Cell 137, 216-233 (2009).

7. Schroder, N. \& Gossler, A. Expression of Notch pathway components in fetal and adult mouse small intestine. Gene Expr. Patterns 2, 247-250 (2002).

8. Yang, Q., Bermingham, N.A., Finegold, M.J. \&Zoghbi, H.Y. Requirement of Math1 for secretory cell lineage commitment in the mouse intestine. Science 294, 2155-2158 (2001).

9. Dahan, S., Rabinowitz, K.M., Martin, A.P., Berin, M.C., Unkeless, J.C. \& Mayer, L. Notch-1 signaling regulates intestinal epithelial barrier function, through interaction with CD4 + T cells, in mice and humans. Gastroenterology 140, 550-559 (2011).

10. Laitman, L.E. \& Dahan, S. Taking inflammatory bowel disease up a Notch. Immunol. Res. 54, 69-74 (2012).

11. Radtke, F., Fasnacht, N. \& Macdonald, H.R. Notch signaling in the immune system. Immunity 32, 14-27 (2010).

12. Amsen, D. et al. Direct regulation of Gata3 expression determines the T helper differentiation potential of Notch. Immunity 27, 89-99 (2007).
13. Amsen, D., Blander, J.M., Lee, G.R., Tanigaki, K., Honjo, T. \& Flavell, R.A. Instruction of distinct CD4 T helper cell fates by different notch ligands on antigen-presenting cells. Cell 117, 515-526 (2004).

14. Palaga, T. et al. Notch signaling is activated by TLR stimulation and regulates macrophage functions. Eur. J. Immunol. 38, 174-183 (2008).

15. Sauma, D., Espejo, P., Ramirez, A., Fierro, A., Rosemblatt, M. \& Bono, M.R. Differential regulation of Notch ligands in dendritic cells upon interaction with T helper cells. Scand. J. Immunol. 74, 62-70 (2011).

16. Dahan, S. et al. Epithelial: lamina propria lymphocyte interactions promote epithelial cell differentiation. Gastroenterology 134, 192-203 (2008).

17. Zhang, Y. et al. Engineering mucosal RNA interference in vivo. Mol. Ther. 14, 336-342 (2006).

18. Schmitt, E.G. et al. IL-10 produced by induced regulatory T cells (iTregs) controls colitis and pathogenic ex-iTregs during immunotherapy. J. Immunol. 189, 5638-5648 (2012).

19. Mukaida, N., Okamoto, S., Ishikawa, Y. \& Matsushima, K. Molecular mechanism of interleukin-8 gene expression. J. Leukoc. Biol. 56, 554-558 (1994).

20. Wongchana, W. \& Palaga, T. Direct regulation of interleukin-6 expression by Notch signaling in macrophages. Cell. Mol. Immunol. 9, 155-162 (2012).

21. Monsalve, E. et al. Notch1 upregulates LPS-induced macrophage activation by increasing NF-kappaB activity. Eur. J. Immunol. 39, 2556-2570 (2009).

22. van Es, J.H. et al. Notch/gamma-secretase inhibition turns proliferative cells in intestinal crypts and adenomas into goblet cells. Nature 435, 959-963 (2005).

23. Obata, Y. et al. Epithelial cell-intrinsic Notch signaling plays an essential role in the maintenance of gut immune homeostasis. J. Immunol. 188, 2427-2436 (2012)

24. Wilson, A., Ferrero, I., MacDonald, H.R. \& Radtke, F. Cutting edge: an essential role for Notch-1 in the development of both thymus-independent and -dependent T cells in the gut. J. Immunol. 165, 5397-5400 (2000).

25. Koduru, S., Kumar, R., Srinivasan, S., Evers, M.B. \& Damodaran, C. Notch1 inhibition by Withaferin-A: a therapeutic target against colon carcinogenesis. Mol. Cancer Ther. 9, 202-210 (2010).

26. Jiao, $X$. et al. Somatic mutations in the Notch, NF-KB, PIK3CA, and Hedgehog pathways in human breast cancers. Genes Chromosomes Cancer 51, 480-489 (2012).

27. Espinosa, L. et al. The Notch/Hes1 pathway sustains NF-kappaB activation through CYLD repression in T cell leukemia. Cancer Cell 18 268-281 (2010).

28. Jefferies, C., Bowie, A., Brady, G., Cooke, E.L., Li, X. \& O'Neill, L.A. Transactivation by the p65 subunit of NF-kappaB in response to interleukin-1 (IL-1) involves MyD88, IL-1 receptor-associated kinase 1, TRAF-6, and Rac1. Mol. Cell. Biol. 21, 4544-4552 (2001).

29. Araki, A. etal. MyD88-deficient mice develop severe intestinal inflammation in dextran sodium sulfate colitis. J. Gastroenterol. 40, 16-23 (2005).

30. Fukata, M. et al. Toll-like receptor- 4 is required for intestinal response to epithelial injury and limiting bacterial translocation in a murine model of acute colitis. Am. J. Physiol. Gastrointest. Liver Physiol. 288, G1055G1065 (2005)

31. Frantz, A.L. et al. Targeted deletion of MyD88 in intestinal epithelial cells results in compromised antibacterial immunity associated with downregulation of polymeric immunoglobulin receptor, mucin-2, and antibacterial peptides. Mucosal Immunol. 5, 501-512 (2012).

32. Zaph, C. et al. Epithelial-cell-intrinsic IKK-beta expression regulates intestinal immune homeostasis. Nature 446, 552-556 (2007).

33. Salim, S.Y. \& Soderholm, J.D. Importance of disrupted intestinal barrier in inflammatory bowel diseases. Inflamm. Bowel Dis. 17, 362-381 (2011).

34. Brabletz, T. et al. Invasion and metastasis in colorectal cancer: epithelialmesenchymal transition, mesenchymal-epithelial transition, stem cells and beta-catenin. Cells Tissues Organs 179, 56-65 (2005).

35. Soler, A.P., Miller, R.D., Laughlin, K.V., Carp, N.Z., Klurfeld, D.M. \& Mullin, J.M. Increased tight junctional permeability is associated with the development of colon cancer. Carcinogenesis 20, 1425-1431 (1999).

36. Grivennikov, S.I. et al. Adenoma-linked barrier defects and microbial products drive IL-23/L-17-mediated tumour growth. Nature 491, 254-258 (2012). 
37. Rabinowitz, K.M. et al. Transforming growth factor beta signaling controls activities of human intestinal CD8(+)T suppressor cells. Gastroenterology 144, 601-12 e1 (2013).

38. Brimnes, J., Allez, M., Dotan, I., Shao, L., Nakazawa, A. \& Mayer, L. Defects in CD8 + regulatory $T$ cells in the lamina propria of patients with inflammatory bowel disease. J. Immunol. 174, 5814-5822 (2005).
39. Ey, B., Eyking, A., Gerken, G., Podolsky, D.K. \& Cario, E. TLR2 mediates gap junctional intercellular communication through connexin43 in intestinal epithelial barrier injury. J. Biol. Chem. 284, 22332-22343 (2009).

40. Totsuka, T. etal. IL-7 Is essential for the development and the persistence of chronic colitis. J. Immunol. 178, 4737-4748 (2007). 